Supporting Information

\title{
Learning Catalyst Design Based on Bias-free Data Set for Oxidative Coupling of Methane
}

Thanh Nhat Nguyen, ${ }^{+}$Sunao Nakanowatari, ${ }^{\dagger}$ Thuy Phuong Nhat Tran,${ }^{\dagger}$ Ashutosh Thakur, ${ }^{\dagger}$ Lauren Takahashi, ${ }^{\ddagger}$ Keisuke Takahashi, ${ }^{\ddagger}$ Toshiaki Taniike ${ }^{*},+$

${ }^{+}$Graduate School of Advanced Science and Technology, Japan Advanced Institute of Science and Technology, 1-1 Asahidai, Nomi, Ishikawa 923-1292, Japan

${ }^{\ddagger}$ Department of Chemistry, Hokkaido University, North 10, West 8, Sapporo 060-8510, Japan

*To whom correspondence should be addressed:taniike@jaist.ac.jp 
Table S1. Catalyst library. The composition, the best performance, and the corresponding conditions are shown.,

\begin{tabular}{|c|c|c|c|c|c|c|c|c|c|c|c|}
\hline No & M1 & M2 & M3 & $\begin{array}{l}\text { Suppo } \\
\text { rt }\end{array}$ & $\begin{array}{l}\mathrm{C}_{2} \\
\text { yield } \\
(\%)\end{array}$ & $\begin{array}{l}\mathrm{CH}_{4} \\
\text { conversi } \\
\text { on }(\%)\end{array}$ & $\begin{array}{l}\mathrm{C}_{2} \\
\text { selectivit } \\
\mathrm{y}(\%)\end{array}$ & $\begin{array}{l}\text { Temp. } \\
\left({ }^{\circ} \mathrm{C}\right)\end{array}$ & $\begin{array}{l}\mathrm{CH}_{4} / \mathrm{O}_{2} \\
(\mathrm{~mol} / \mathrm{mol})\end{array}$ & $\begin{array}{l}\text { Total } \\
\text { flow } \\
(\mathrm{mL} / \mathrm{mi} \\
\mathrm{n})\end{array}$ & $\begin{array}{l}\mathrm{P}_{\mathrm{Ar}} \\
(\mathrm{atm})\end{array}$ \\
\hline 1 & $\mathrm{Li}$ & $\mathrm{K}$ & $\mathrm{Mn}$ & $\mathrm{MgO}$ & 8.2 & 29.0 & 28.3 & 800 & 2 & 10 & 0.15 \\
\hline 2 & $\mathrm{Li}$ & $\mathrm{Fe}$ & $\mathrm{Tb}$ & $\mathrm{MgO}$ & 4.7 & 24.7 & 19.1 & 850 & 2 & 10 & 0.40 \\
\hline 3 & $\mathrm{Li}$ & $\mathrm{Fe}$ & Hf & $\mathrm{MgO}$ & 2.5 & 28.1 & 8.8 & 900 & 2 & 10 & 0.15 \\
\hline 4 & $\mathrm{Na}$ & $\mathrm{Na}$ & none & $\mathrm{MgO}$ & 7.4 & 34.0 & 21.7 & 850 & 2 & 20 & 0.15 \\
\hline 5 & $\mathrm{Na}$ & $\mathrm{Ni}$ & Y & $\mathrm{MgO}$ & 1.0 & 24.6 & 4.1 & 750 & 6 & 20 & 0.70 \\
\hline 6 & $\mathrm{Na}$ & $\mathrm{Ce}$ & $\mathrm{Tb}$ & $\mathrm{MgO}$ & 3.8 & 32.1 & 11.9 & 900 & 6 & 20 & 0.15 \\
\hline 7 & $\mathrm{Na}$ & $\mathrm{Tb}$ & $\mathrm{Hf}$ & $\mathrm{MgO}$ & 8.6 & 30.9 & 27.7 & 800 & 2 & 20 & 0.15 \\
\hline 8 & $\mathrm{Na}$ & $\mathrm{Eu}$ & $\mathrm{Hf}$ & $\mathrm{MgO}$ & 16.6 & 35.6 & 46.7 & 750 & 2 & 10 & 0.15 \\
\hline 9 & $\mathrm{Mg}$ & $\mathrm{Ca}$ & $\mathrm{Zn}$ & $\mathrm{MgO}$ & 2.1 & 24.0 & 8.9 & 850 & 4 & 15 & 0.15 \\
\hline 10 & $\mathrm{Mg}$ & $\mathrm{Mn}$ & none & $\mathrm{MgO}$ & 7.9 & 27.1 & 29.0 & 850 & 2 & 20 & 0.15 \\
\hline 11 & $\mathrm{Mg}$ & $\mathrm{Zn}$ & $\mathrm{Eu}$ & $\mathrm{MgO}$ & 15.5 & 40.9 & 37.9 & 700 & 2 & 20 & 0.15 \\
\hline 12 & $\mathrm{Mg}$ & $\mathrm{Nd}$ & none & $\mathrm{MgO}$ & 9.5 & 35.5 & 26.8 & 850 & 2 & 20 & 0.70 \\
\hline 13 & $\mathrm{Ca}$ & $\mathrm{Zr}$ & $\mathrm{La}$ & $\mathrm{MgO}$ & 16.1 & 33.8 & 47.6 & 750 & 2 & 20 & 0.15 \\
\hline 14 & $\mathrm{Ti}$ & $\mathrm{Ni}$ & Cs & $\mathrm{MgO}$ & 1.6 & 33.2 & 4.8 & 800 & 2 & 20 & 0.40 \\
\hline 15 & $\mathrm{Ti}$ & $\mathrm{V}$ & $\mathrm{Ba}$ & $\mathrm{MgO}$ & 8.2 & 37.7 & 21.8 & 900 & 2 & 15 & 0.70 \\
\hline 16 & $\mathrm{~V}$ & $\mathrm{Fe}$ & $\mathrm{Ni}$ & $\mathrm{MgO}$ & 7.6 & 34.3 & 22.0 & 800 & 2 & 15 & 0.15 \\
\hline 17 & $\mathrm{~V}$ & Mo & $\mathrm{Pd}$ & $\mathrm{MgO}$ & 0.7 & 26.5 & 2.6 & 900 & 6 & 15 & 0.70 \\
\hline 18 & $\mathrm{~V}$ & $\mathrm{~Tb}$ & W & $\mathrm{MgO}$ & 6.7 & 33.6 & 19.9 & 850 & 2 & 15 & 0.15 \\
\hline 19 & $\mathrm{Fe}$ & $\mathrm{Y}$ & $\mathrm{Nd}$ & $\mathrm{MgO}$ & 3.6 & 21.5 & 16.9 & 800 & 2 & 20 & 0.15 \\
\hline 20 & $\mathrm{Fe}$ & $\mathrm{Zr}$ & none & $\mathrm{MgO}$ & 7.1 & 37.8 & 18.7 & 850 & 2 & 20 & 0.15 \\
\hline 21 & $\mathrm{Fe}$ & $\mathrm{Ce}$ & $\mathrm{Tb}$ & $\mathrm{MgO}$ & 6.2 & 25.0 & 25.0 & 800 & 2 & 10 & 0.15 \\
\hline 22 & $\mathrm{Fe}$ & $\mathrm{La}$ & $\mathrm{Hf}$ & $\mathrm{MgO}$ & 6.5 & 29.3 & 22.2 & 850 & 2 & 15 & 0.15 \\
\hline 23 & Co & $\mathrm{Zn}$ & $\mathrm{Sr}$ & $\mathrm{MgO}$ & 1.0 & 31.0 & 3.2 & 850 & 6 & 20 & 0.15 \\
\hline 24 & Co & $\mathrm{Zn}$ & $\mathrm{Tb}$ & $\mathrm{MgO}$ & 5.7 & 36.6 & 15.6 & 900 & 2 & 20 & 0.15 \\
\hline 25 & Co & $\mathrm{Y}$ & $\mathrm{Hf}$ & $\mathrm{MgO}$ & 7.0 & 26.6 & 26.4 & 700 & 2 & 20 & 0.15 \\
\hline 26 & $\mathrm{Ni}$ & $\mathrm{Y}$ & $\mathrm{Tb}$ & $\mathrm{MgO}$ & 5.6 & 18.3 & 30.7 & 900 & 4 & 20 & 0.70 \\
\hline 27 & $\mathrm{Y}$ & $\mathrm{La}$ & $\mathrm{Ce}$ & $\mathrm{MgO}$ & 12.1 & 37.8 & 32.1 & 700 & 2 & 20 & 0.15 \\
\hline 28 & Mo & Cs & $\mathrm{La}$ & $\mathrm{MgO}$ & 8.5 & 31.8 & 26.8 & 850 & 2 & 20 & 0.40 \\
\hline 29 & Mo & $\mathrm{Ce}$ & $\mathrm{Tb}$ & $\mathrm{MgO}$ & 7.3 & 33.2 & 22.0 & 800 & 2 & 20 & 0.15 \\
\hline 30 & $\mathrm{Li}$ & $\mathrm{Mg}$ & Hf & $\mathrm{Al}_{2} \mathrm{O}_{3}$ & 3.2 & 40.4 & 7.9 & 850 & 2 & 15 & 0.15 \\
\hline 31 & $\mathrm{Li}$ & $\mathrm{Ca}$ & $\mathrm{Mn}$ & $\mathrm{Al}_{2} \mathrm{O}_{3}$ & 7.2 & 38.4 & 18.8 & 850 & 2 & 20 & 0.15 \\
\hline 32 & $\mathrm{Li}$ & $\mathrm{Ti}$ & $\mathrm{La}$ & $\mathrm{Al}_{2} \mathrm{O}_{3}$ & 15.5 & 37.4 & 41.4 & 850 & 2 & 15 & 0.70 \\
\hline 33 & $\mathrm{Li}$ & $\mathrm{Fe}$ & none & $\mathrm{Al}_{2} \mathrm{O}_{3}$ & 9.2 & 32.2 & 28.4 & 850 & 2 & 10 & 0.70 \\
\hline 34 & $\mathrm{Li}$ & $\mathrm{Hf}$ & none & $\mathrm{Al}_{2} \mathrm{O}_{3}$ & 4.9 & 35.2 & 14.0 & 900 & 2 & 20 & 0.40 \\
\hline 35 & $\mathrm{Na}$ & $\mathrm{K}$ & Hf & $\mathrm{Al}_{2} \mathrm{O}_{3}$ & 8.2 & 32.0 & 25.7 & 850 & 2 & 10 & 0.40 \\
\hline 36 & $\mathrm{Na}$ & $\mathrm{Ti}$ & $\mathrm{Cu}$ & $\mathrm{Al}_{2} \mathrm{O}_{3}$ & 11.5 & 20.7 & 55.9 & 850 & 4 & 20 & 0.40 \\
\hline 37 & $\mathrm{Na}$ & $\mathrm{Mn}$ & $\mathrm{Ni}$ & $\mathrm{Al}_{2} \mathrm{O}_{3}$ & 1.2 & 26.6 & 4.5 & 850 & 6 & 20 & 0.70 \\
\hline 38 & $\mathrm{Na}$ & Co & $\mathrm{Ni}$ & $\mathrm{Al}_{2} \mathrm{O}_{3}$ & 7.8 & 28.5 & 27.2 & 800 & 2 & 10 & 0.15 \\
\hline 39 & $\mathrm{Na}$ & Co & none & $\mathrm{Al}_{2} \mathrm{O}_{3}$ & 9.3 & 35.7 & 26.2 & 850 & 2 & 10 & 0.70 \\
\hline 40 & $\mathrm{Na}$ & $\mathrm{Cu}$ & $\mathrm{Ce}$ & $\mathrm{Al}_{2} \mathrm{O}_{3}$ & 3.2 & 27.4 & 11.5 & 900 & 2 & 10 & 0.15 \\
\hline 41 & $\mathrm{Mg}$ & $\mathrm{Mn}$ & Cs & $\mathrm{Al}_{2} \mathrm{O}_{3}$ & 8.6 & 32.8 & 26.2 & 900 & 2 & 20 & 0.70 \\
\hline 42 & $\mathrm{Mg}$ & Co & $\mathrm{Ba}$ & $\mathrm{Al}_{2} \mathrm{O}_{3}$ & 2.7 & 26.9 & 10.0 & 900 & 2 & 10 & 0.15 \\
\hline 43 & $\mathrm{Mg}$ & $\mathrm{La}$ & $\mathrm{Nd}$ & $\mathrm{Al}_{2} \mathrm{O}_{3}$ & 8.2 & 37.7 & 21.6 & 850 & 2 & 20 & 0.15 \\
\hline 44 & $\mathrm{~K}$ & $\mathrm{Mn}$ & $\mathrm{Ni}$ & $\mathrm{Al}_{2} \mathrm{O}_{3}$ & 1.5 & 26.4 & 5.8 & 900 & 6 & 15 & 0.70 \\
\hline 45 & $\mathrm{~K}$ & Co & Cs & $\mathrm{Al}_{2} \mathrm{O}_{3}$ & 9.3 & 34.2 & 27.4 & 850 & 2 & 15 & 0.70 \\
\hline 46 & $\mathrm{~K}$ & $\mathrm{Y}$ & $\mathrm{Hf}$ & $\mathrm{Al}_{2} \mathrm{O}_{3}$ & 10.8 & 20.5 & 52.4 & 850 & 4 & 15 & 0.70 \\
\hline 47 & $\mathrm{~K}$ & $\mathrm{La}$ & $\mathrm{W}$ & $\mathrm{Al}_{2} \mathrm{O}_{3}$ & 4.7 & 24.0 & 19.7 & 800 & 2 & 20 & 0.40 \\
\hline 48 & $\mathrm{Ti}$ & $\mathrm{Mn}$ & Y & $\mathrm{Al}_{2} \mathrm{O}_{3}$ & 10.4 & 32.4 & 32.2 & 850 & 2 & 20 & 0.15 \\
\hline 49 & $\mathrm{Ti}$ & $\mathrm{Fe}$ & Mo & $\mathrm{Al}_{2} \mathrm{O}_{3}$ & 9.4 & 36.8 & 25.5 & 850 & 2 & 20 & 0.40 \\
\hline 50 & $\mathrm{Ti}$ & Cs & $\mathrm{La}$ & $\mathrm{Al}_{2} \mathrm{O}_{3}$ & 7.6 & 30.7 & 24.9 & 800 & 2 & 20 & 0.40 \\
\hline 51 & $\mathrm{~V}$ & $\mathrm{Cu}$ & $\mathrm{Ba}$ & $\mathrm{Al}_{2} \mathrm{O}_{3}$ & 1.8 & 37.7 & 4.7 & 900 & 2 & 20 & 0.15 \\
\hline 52 & $\mathrm{Fe}$ & $\mathrm{Pd}$ & $\mathrm{Nd}$ & $\mathrm{Al}_{2} \mathrm{O}_{3}$ & 11.6 & 20.3 & 57.3 & 850 & 4 & 20 & 0.15 \\
\hline 53 & $\mathrm{Fe}$ & $\mathrm{Ba}$ & none & $\mathrm{Al}_{2} \mathrm{O}_{3}$ & 4.9 & 33.0 & 14.7 & 850 & 2 & 20 & 0.15 \\
\hline 54 & $\mathrm{Fe}$ & $\mathrm{La}$ & $\mathrm{W}$ & $\mathrm{Al}_{2} \mathrm{O}_{3}$ & 2.7 & 36.3 & 7.5 & 850 & 2 & 20 & 0.15 \\
\hline 55 & Co & $\mathrm{Ni}$ & $\mathrm{Zr}$ & $\mathrm{Al}_{2} \mathrm{O}_{3}$ & 1.3 & 32.3 & 4.1 & 900 & 2 & 20 & 0.15 \\
\hline 56 & Co & $\mathrm{Zn}$ & $\mathrm{Nd}$ & $\mathrm{Al}_{2} \mathrm{O}_{3}$ & 10.2 & 36.1 & 28.3 & 850 & 2 & 20 & 0.40 \\
\hline
\end{tabular}




\begin{tabular}{|c|c|c|c|c|c|c|c|c|c|c|c|}
\hline 57 & Co & $\mathrm{Y}$ & $\mathrm{Tb}$ & $\mathrm{Al}_{2} \mathrm{O}_{3}$ & 5.9 & 35.8 & 16.5 & 800 & 2 & 20 & 0.15 \\
\hline 58 & $\mathrm{Ni}$ & $\mathrm{Cu}$ & $\mathrm{Zr}$ & $\mathrm{Al}_{2} \mathrm{O}_{3}$ & 1.6 & 37.8 & 4.2 & 750 & 4 & 20 & 0.70 \\
\hline 59 & $\mathrm{Ni}$ & $\mathrm{Zn}$ & $\mathrm{La}$ & $\mathrm{Al}_{2} \mathrm{O}_{3}$ & 1.0 & 25.5 & 3.8 & 750 & 6 & 10 & 0.70 \\
\hline 60 & $\mathrm{Cu}$ & Y & $\mathrm{Ce}$ & $\mathrm{Al}_{2} \mathrm{O}_{3}$ & 1.7 & 20.0 & 8.4 & 900 & 6 & 20 & 0.15 \\
\hline 61 & $\mathrm{Zn}$ & $\mathrm{Pd}$ & Cs & $\mathrm{Al}_{2} \mathrm{O}_{3}$ & 2.0 & 26.9 & 7.6 & 850 & 6 & 15 & 0.70 \\
\hline 62 & $\mathrm{Sr}$ & $\mathrm{Pd}$ & none & $\mathrm{Al}_{2} \mathrm{O}_{3}$ & 9.1 & 21.0 & 43.1 & 850 & 4 & 10 & 0.15 \\
\hline 63 & $\mathrm{Y}$ & $\mathrm{Pd}$ & $\mathrm{Ba}$ & $\mathrm{Al}_{2} \mathrm{O}_{3}$ & 9.4 & 33.6 & 27.9 & 850 & 2 & 20 & 0.40 \\
\hline 64 & $\mathrm{Zr}$ & Cs & $\mathrm{La}$ & $\mathrm{Al}_{2} \mathrm{O}_{3}$ & 9.3 & 31.2 & 29.7 & 850 & 2 & 15 & 0.70 \\
\hline 65 & $\mathrm{Ba}$ & $\mathrm{La}$ & W & $\mathrm{Al}_{2} \mathrm{O}_{3}$ & 3.5 & 22.5 & 15.6 & 800 & 2 & 20 & 0.40 \\
\hline 66 & $\mathrm{Li}$ & $\mathrm{K}$ & $\mathrm{Co}$ & $\mathrm{SiO}_{2}$ & 10.4 & 30.3 & 34.4 & 850 & 2 & 10 & 0.70 \\
\hline 67 & $\mathrm{Li}$ & $\mathrm{Fe}$ & W & $\mathrm{SiO}_{2}$ & 6.5 & 36.9 & 17.7 & 900 & 2 & 20 & 0.40 \\
\hline 68 & $\mathrm{Li}$ & $\mathrm{Ni}$ & none & $\mathrm{SiO}_{2}$ & 8.1 & 34.9 & 23.3 & 850 & 2 & 20 & 0.40 \\
\hline 69 & $\mathrm{Li}$ & $\mathrm{Nd}$ & $\mathrm{Tb}$ & $\mathrm{SiO}_{2}$ & 8.4 & 26.4 & 31.8 & 850 & 2 & 20 & 0.40 \\
\hline 70 & $\mathrm{Li}$ & $\mathrm{Ni}$ & Hf & $\mathrm{SiO}_{2}$ & 11.2 & 33.1 & 33.7 & 850 & 2 & 20 & 0.40 \\
\hline 71 & $\mathrm{Na}$ & $\mathrm{Sr}$ & Mo & $\mathrm{SiO}_{2}$ & 12.9 & 36.6 & 35.2 & 850 & 2 & 10 & 0.70 \\
\hline 72 & $\mathrm{Na}$ & $\mathrm{Ce}$ & $\mathrm{Tb}$ & $\mathrm{SiO}_{2}$ & 8.9 & 26.8 & 33.3 & 850 & 2 & 20 & 0.40 \\
\hline 73 & $\mathrm{Mg}$ & $\mathrm{V}$ & $\mathrm{Pd}$ & $\mathrm{SiO}_{2}$ & n.d. & n.d. & n.d. & n.d. & n.d. & n.d. & n.d. \\
\hline 74 & $\mathrm{Mg}$ & $\mathrm{Cu}$ & $\mathrm{Sr}$ & $\mathrm{SiO}_{2}$ & 7.3 & 27.1 & 27.0 & 800 & 2 & 10 & 0.15 \\
\hline 75 & $\mathrm{Mg}$ & $\mathrm{Zn}$ & $\mathrm{Eu}$ & $\mathrm{SiO}_{2}$ & 7.6 & 43.2 & 17.6 & 850 & 2 & 20 & 0.15 \\
\hline 76 & $\mathrm{~K}$ & $\mathrm{Mn}$ & $\mathrm{La}$ & $\mathrm{SiO}_{2}$ & 8.1 & 36.5 & 22.2 & 850 & 2 & 20 & 0.40 \\
\hline 77 & $\mathrm{~K}$ & $\mathrm{Zr}$ & $\mathrm{Tb}$ & $\mathrm{SiO}_{2}$ & 7.7 & 15.3 & 50.4 & 900 & 4 & 20 & 0.15 \\
\hline 78 & $\mathrm{Ca}$ & $\mathrm{Ti}$ & $\mathrm{Cu}$ & $\mathrm{SiO}_{2}$ & 9.7 & 34.4 & 28.2 & 900 & 2 & 20 & 0.70 \\
\hline 79 & $\mathrm{Ti}$ & $\mathrm{Sr}$ & $\mathrm{La}$ & $\mathrm{SiO}_{2}$ & 8.4 & 29.3 & 28.7 & 850 & 2 & 20 & 0.15 \\
\hline 80 & $\mathrm{Ti}$ & Mo & $\mathrm{Nd}$ & $\mathrm{SiO}_{2}$ & 4.5 & 30.8 & 14.8 & 900 & 6 & 20 & 0.15 \\
\hline 81 & $\mathrm{~V}$ & $\mathrm{Pd}$ & $\mathrm{Nd}$ & $\mathrm{SiO}_{2}$ & n.d. & n.d. & n.d. & n.d. & n.d. & n.d. & n.d. \\
\hline 82 & $\mathrm{~V}$ & Cs & $\mathrm{Nd}$ & $\mathrm{SiO}_{2}$ & 8.0 & 31.1 & 25.8 & 850 & 2 & 20 & 0.15 \\
\hline 83 & $\mathrm{Mn}$ & $\mathrm{Fe}$ & $\mathrm{Cu}$ & $\mathrm{SiO}_{2}$ & 7.4 & 34.6 & 21.3 & 850 & 2 & 20 & 0.15 \\
\hline 84 & $\mathrm{Mn}$ & $\mathrm{Zn}$ & $\mathrm{Sr}$ & $\mathrm{SiO}_{2}$ & 10.4 & 31.8 & 32.7 & 900 & 2 & 20 & 0.70 \\
\hline 85 & Co & $\mathrm{Ni}$ & $\mathrm{Cu}$ & $\mathrm{SiO}_{2}$ & 6.1 & 33.9 & 18.1 & 850 & 2 & 20 & 0.40 \\
\hline 86 & Co & $\mathrm{Y}$ & $\mathrm{Nd}$ & $\mathrm{SiO}_{2}$ & 8.7 & 23.5 & 37.1 & 800 & 2 & 20 & 0.15 \\
\hline 87 & $\mathrm{Ni}$ & W & none & $\mathrm{SiO}_{2}$ & 0.7 & 2.2 & 30.2 & 700 & 2 & 15 & 0.70 \\
\hline 88 & $\mathrm{Ni}$ & $\mathrm{Ce}$ & $\mathrm{Nd}$ & $\mathrm{SiO}_{2}$ & 8.3 & 26.9 & 30.9 & 850 & 2 & 10 & 0.70 \\
\hline 89 & $\mathrm{Cu}$ & $\mathrm{La}$ & $\mathrm{Tb}$ & $\mathrm{SiO}_{2}$ & 3.9 & 37.0 & 10.5 & 900 & 2 & 20 & 0.40 \\
\hline 90 & $\mathrm{Cu}$ & none & none & $\mathrm{SiO}_{2}$ & 8.1 & 34.3 & 23.6 & 800 & 2 & 10 & 0.15 \\
\hline 91 & $\mathrm{Zn}$ & $\mathrm{Sr}$ & $\mathrm{Pd}$ & $\mathrm{SiO}_{2}$ & 1.2 & 43.5 & 2.8 & 850 & 2 & 20 & 0.70 \\
\hline 92 & $\mathrm{Zr}$ & Cs & Cs & $\mathrm{SiO}_{2}$ & 2.5 & 18.0 & 13.8 & 900 & 6 & 15 & 0.15 \\
\hline 93 & Mo & $\mathrm{Ba}$ & none & $\mathrm{SiO}_{2}$ & 8.5 & 32.8 & 25.9 & 850 & 2 & 20 & 0.40 \\
\hline 94 & Cs & $\mathrm{Ba}$ & $\mathrm{Ce}$ & $\mathrm{SiO}_{2}$ & 7.8 & 23.2 & 33.6 & 850 & 2 & 15 & 0.70 \\
\hline 95 & $\mathrm{Li}$ & $\mathrm{V}$ & $\mathrm{Eu}$ & $\mathrm{CaO}$ & 13.6 & 36.6 & 37.2 & 850 & 2 & 20 & 0.70 \\
\hline 96 & $\mathrm{Li}$ & Co & Mo & $\mathrm{CaO}$ & 4.8 & 25.4 & 18.9 & 750 & 2 & 10 & 0.15 \\
\hline 97 & $\mathrm{Li}$ & $\mathrm{Cu}$ & Cs & $\mathrm{CaO}$ & 5.6 & 31.0 & 18.1 & 900 & 2 & 20 & 0.15 \\
\hline 98 & $\mathrm{Na}$ & $\mathrm{Mg}$ & Hf & $\mathrm{CaO}$ & 14.3 & 33.6 & 42.5 & 750 & 2 & 15 & 0.40 \\
\hline 99 & $\mathrm{Na}$ & $\mathrm{Ti}$ & $\mathrm{Ce}$ & $\mathrm{CaO}$ & 11.9 & 31.6 & 37.6 & 700 & 2 & 20 & 0.15 \\
\hline 100 & $\mathrm{Na}$ & $\mathrm{Mn}$ & $\mathrm{La}$ & $\mathrm{CaO}$ & 6.7 & 33.3 & 20.2 & 800 & 2 & 20 & 0.15 \\
\hline 101 & $\mathrm{Na}$ & $\mathrm{Cu}$ & Hf & $\mathrm{CaO}$ & 4.2 & 25.6 & 16.3 & 800 & 2 & 20 & 0.15 \\
\hline 102 & $\mathrm{Mg}$ & Co & $\mathrm{Ce}$ & $\mathrm{CaO}$ & 8.0 & 33.5 & 23.9 & 800 & 2 & 20 & 0.15 \\
\hline 103 & $\mathrm{Mg}$ & $\mathrm{Cu}$ & $\mathrm{Eu}$ & $\mathrm{CaO}$ & 4.6 & 37.7 & 12.2 & 900 & 2 & 20 & 0.15 \\
\hline 104 & $\mathrm{Mg}$ & $\mathrm{Sr}$ & $\mathrm{Y}$ & $\mathrm{CaO}$ & 14.4 & 35.6 & 40.5 & 800 & 2 & 20 & 0.40 \\
\hline 105 & $\mathrm{Mg}$ & $\mathrm{Sr}$ & $\mathrm{Ba}$ & $\mathrm{CaO}$ & 17.4 & 39.6 & 44.1 & 800 & 2 & 20 & 0.70 \\
\hline 106 & $\mathrm{Mg}$ & $\mathrm{Zr}$ & $\mathrm{Hf}$ & $\mathrm{CaO}$ & 15.9 & 36.6 & 43.6 & 750 & 2 & 20 & 0.15 \\
\hline 107 & $\mathrm{~K}$ & $\mathrm{Sr}$ & $\mathrm{Y}$ & $\mathrm{CaO}$ & 9.9 & 33.2 & 29.8 & 700 & 2 & 15 & 0.40 \\
\hline 108 & $\mathrm{~K}$ & $\mathrm{Y}$ & $\mathrm{Eu}$ & $\mathrm{CaO}$ & 13.1 & 40.4 & 32.3 & 750 & 2 & 20 & 0.15 \\
\hline 109 & $\mathrm{Ca}$ & $\mathrm{Mn}$ & none & $\mathrm{CaO}$ & 7.6 & 28.4 & 26.6 & 850 & 2 & 15 & 0.15 \\
\hline 110 & $\mathrm{Ca}$ & $\mathrm{Nd}$ & $\mathrm{Tb}$ & $\mathrm{CaO}$ & 7.2 & 38.4 & 18.8 & 850 & 2 & 20 & 0.15 \\
\hline 111 & $\mathrm{Ti}$ & $\mathrm{Fe}$ & $\mathrm{Sr}$ & $\mathrm{CaO}$ & 10.3 & 31.2 & 32.9 & 700 & 2 & 20 & 0.15 \\
\hline 112 & $\mathrm{Ti}$ & $\mathrm{Zn}$ & $\mathrm{Tb}$ & $\mathrm{CaO}$ & 12.5 & 36.1 & 34.6 & 700 & 2 & 20 & 0.15 \\
\hline 113 & $\mathrm{Ti}$ & Cs & $\mathrm{Ce}$ & $\mathrm{CaO}$ & 15.4 & 33.2 & 46.3 & 700 & 2 & 15 & 0.15 \\
\hline 114 & $\mathrm{~V}$ & $\mathrm{Pd}$ & W & $\mathrm{CaO}$ & 0.7 & 30.8 & 2.4 & 900 & 6 & 10 & 0.70 \\
\hline 115 & $\mathrm{~V}$ & $\mathrm{Ce}$ & $\mathrm{Hf}$ & $\mathrm{CaO}$ & 12.3 & 31.1 & 39.4 & 750 & 2 & 15 & 0.15 \\
\hline 116 & $\mathrm{Mn}$ & $\mathrm{Tb}$ & $\mathrm{Hf}$ & $\mathrm{CaO}$ & 8.9 & 27.9 & 31.8 & 800 & 2 & 10 & 0.15 \\
\hline 117 & $\mathrm{Mn}$ & $\mathrm{Fe}$ & $\mathrm{Hf}$ & $\mathrm{CaO}$ & 7.7 & 24.5 & 31.4 & 800 & 2 & 15 & 0.15 \\
\hline 118 & $\mathrm{Fe}$ & $\mathrm{Y}$ & $\mathrm{Tb}$ & $\mathrm{CaO}$ & 9.4 & 30.4 & 30.8 & 750 & 2 & 10 & 0.15 \\
\hline
\end{tabular}




\begin{tabular}{|c|c|c|c|c|c|c|c|c|c|c|c|}
\hline 119 & $\mathrm{Fe}$ & $\mathrm{Ba}$ & $\mathrm{Ce}$ & $\mathrm{CaO}$ & 11.6 & 37.6 & 30.9 & 750 & 2 & 20 & 0.15 \\
\hline 120 & Co & $\mathrm{Cu}$ & $\mathrm{Nd}$ & $\mathrm{CaO}$ & 3.2 & 34.4 & 9.3 & 900 & 2 & 10 & 0.15 \\
\hline 121 & $\mathrm{Sr}$ & Cs & $\mathrm{La}$ & $\mathrm{CaO}$ & 8.0 & 17.2 & 46.3 & 750 & 4 & 20 & 0.15 \\
\hline 122 & $\mathrm{Sr}$ & $\mathrm{Pd}$ & W & $\mathrm{CaO}$ & 1.0 & 33.4 & 3.0 & 900 & 6 & 15 & 0.70 \\
\hline 123 & $\mathrm{Y}$ & Mo & $\mathrm{Nd}$ & $\mathrm{CaO}$ & 13.2 & 40.7 & 32.5 & 800 & 2 & 20 & 0.40 \\
\hline 124 & $\mathrm{Ba}$ & $\mathrm{Eu}$ & none & $\mathrm{CaO}$ & 17.0 & 42.8 & 39.6 & 750 & 2 & 20 & 0.15 \\
\hline 125 & $\mathrm{Li}$ & $\mathrm{Ti}$ & V & $\mathrm{TiO}_{2}$ & 8.4 & 34.2 & 24.5 & 850 & 2 & 20 & 0.15 \\
\hline 126 & $\mathrm{Li}$ & $\mathrm{Fe}$ & $\mathrm{Ce}$ & $\mathrm{TiO}_{2}$ & 11.6 & 38.2 & 30.3 & 900 & 2 & 20 & 0.70 \\
\hline 127 & $\mathrm{Li}$ & $\mathrm{Zn}$ & $\mathrm{Pd}$ & $\mathrm{TiO}_{2}$ & 7.5 & 35.5 & 21.1 & 850 & 2 & 20 & 0.15 \\
\hline 128 & $\mathrm{Na}$ & $\mathrm{Fe}$ & $\mathrm{Pd}$ & $\mathrm{TiO}_{2}$ & 2.1 & 33.7 & 6.2 & 850 & 2 & 20 & 0.15 \\
\hline 129 & $\mathrm{Na}$ & Co & $\mathrm{Pd}$ & $\mathrm{TiO}_{2}$ & n.d. & n.d. & n.d. & n.d. & n.d. & n.d. & n.d. \\
\hline 130 & $\mathrm{Mg}$ & $\mathrm{Mn}$ & $\mathrm{Ce}$ & $\mathrm{TiO}_{2}$ & 14.2 & 37.4 & 38.0 & 850 & 2 & 15 & 0.70 \\
\hline 131 & $\mathrm{Mg}$ & $\mathrm{Sr}$ & $\mathrm{Tb}$ & $\mathrm{TiO}_{2}$ & 10.3 & 36.4 & 28.4 & 900 & 2 & 20 & 0.70 \\
\hline 132 & $\mathrm{Mg}$ & $\mathrm{Ti}$ & $\mathrm{La}$ & $\mathrm{TiO}_{2}$ & 12.0 & 32.5 & 37.0 & 850 & 2 & 15 & 0.70 \\
\hline 133 & $\mathrm{Ca}$ & $\mathrm{V}$ & $\mathrm{Y}$ & $\mathrm{TiO}_{2}$ & 8.6 & 29.1 & 29.5 & 800 & 2 & 20 & 0.40 \\
\hline 134 & $\mathrm{Ti}$ & $\mathrm{Zn}$ & $\mathrm{La}$ & $\mathrm{TiO}_{2}$ & 10.4 & 28.5 & 36.4 & 800 & 2 & 20 & 0.40 \\
\hline 135 & $\mathrm{Ti}$ & $\mathrm{Zr}$ & $\mathrm{Ce}$ & $\mathrm{TiO}_{2}$ & 15.2 & 38.4 & 39.6 & 800 & 2 & 20 & 0.40 \\
\hline 136 & $\mathrm{Ti}$ & Cs & $\mathrm{W}$ & $\mathrm{TiO}_{2}$ & 16.6 & 37.8 & 43.8 & 750 & 2 & 20 & 0.40 \\
\hline 137 & $\mathrm{Ti}$ & Y & $\mathrm{Ce}$ & $\mathrm{TiO}_{2}$ & 1.3 & 24.0 & 5.5 & 800 & 2 & 10 & 0.15 \\
\hline 138 & V & Co & $\mathrm{La}$ & $\mathrm{TiO}_{2}$ & 8.1 & 30.8 & 26.4 & 800 & 2 & 10 & 0.15 \\
\hline 139 & $\mathrm{~V}$ & Co & $\mathrm{Ce}$ & $\mathrm{TiO}_{2}$ & 5.5 & 30.8 & 17.8 & 700 & 2 & 10 & 0.15 \\
\hline 140 & $\mathrm{~V}$ & $\mathrm{Ni}$ & $\mathrm{Zn}$ & $\mathrm{TiO}_{2}$ & 6.1 & 30.1 & 20.3 & 800 & 2 & 10 & 0.15 \\
\hline 141 & $\mathrm{~V}$ & $\mathrm{Pd}$ & none & $\mathrm{TiO}_{2}$ & n.d. & n.d. & n.d. & n.d. & n.d. & n.d. & n.d. \\
\hline 142 & $\mathrm{Mn}$ & $\mathrm{Fe}$ & $\mathrm{Nd}$ & $\mathrm{TiO}_{2}$ & 9.4 & 30.4 & 31.0 & 750 & 2 & 20 & 0.40 \\
\hline 143 & $\mathrm{Mn}$ & $\mathrm{Cu}$ & $\mathrm{Sr}$ & $\mathrm{TiO}_{2}$ & 8.2 & 26.6 & 30.7 & 850 & 4 & 20 & 0.15 \\
\hline 144 & $\mathrm{Mn}$ & $\mathrm{Nd}$ & $\mathrm{W}$ & $\mathrm{TiO}_{2}$ & 7.9 & 37.0 & 21.4 & 900 & 2 & 20 & 0.40 \\
\hline 145 & $\mathrm{Fe}$ & $\mathrm{Fe}$ & $\mathrm{Ni}$ & $\mathrm{TiO}_{2}$ & n.d. & n.d. & n.d. & n.d. & n.d. & n.d. & n.d. \\
\hline 146 & $\mathrm{Fe}$ & $\mathrm{Cu}$ & $\mathrm{Sr}$ & $\mathrm{TiO}_{2}$ & 8.2 & 39.6 & 20.7 & 850 & 2 & 20 & 0.15 \\
\hline 147 & $\mathrm{Fe}$ & $\mathrm{Zn}$ & W & $\mathrm{TiO}_{2}$ & 15.4 & 31.3 & 49.2 & 850 & 4 & 20 & 0.15 \\
\hline 148 & $\mathrm{Fe}$ & $\mathrm{Sr}$ & Mo & $\mathrm{TiO}_{2}$ & 8.3 & 31.2 & 26.4 & 800 & 2 & 10 & 0.15 \\
\hline 149 & Co & $\mathrm{Sr}$ & $\mathrm{Hf}$ & $\mathrm{TiO}_{2}$ & 13.7 & 37.3 & 36.8 & 800 & 2 & 20 & 0.15 \\
\hline 150 & $\mathrm{Ni}$ & $\mathrm{Zn}$ & $\mathrm{La}$ & $\mathrm{TiO}_{2}$ & 2.1 & 14.9 & 14.3 & 750 & 4 & 10 & 0.70 \\
\hline 151 & $\mathrm{Ni}$ & $\mathrm{Ce}$ & W & $\mathrm{TiO}_{2}$ & 9.2 & 30.4 & 30.3 & 750 & 2 & 10 & 0.15 \\
\hline 152 & $\mathrm{Ni}$ & Mo & $\mathrm{Eu}$ & $\mathrm{TiO}_{2}$ & 4.2 & 25.4 & 16.5 & 850 & 2 & 20 & 0.70 \\
\hline 153 & $\mathrm{Cu}$ & $\mathrm{Zr}$ & $\mathrm{Tb}$ & $\mathrm{TiO}_{2}$ & 7.4 & 37.8 & 19.6 & 850 & 2 & 20 & 0.15 \\
\hline 154 & $\mathrm{Cu}$ & Mo & $\mathrm{Ce}$ & $\mathrm{TiO}_{2}$ & 15.3 & 38.7 & 39.5 & 800 & 2 & 20 & 0.40 \\
\hline 155 & $\mathrm{Cu}$ & $\mathrm{La}$ & $\mathrm{Tb}$ & $\mathrm{TiO}_{2}$ & 10.3 & 32.5 & 31.7 & 850 & 2 & 20 & 0.40 \\
\hline 156 & $\mathrm{Cu}$ & $\mathrm{Eu}$ & $\mathrm{Eu}$ & $\mathrm{TiO}_{2}$ & 5.9 & 35.8 & 16.5 & 800 & 2 & 20 & 0.15 \\
\hline 157 & $\mathrm{Zn}$ & $\mathrm{Zn}$ & $\mathrm{Nd}$ & $\mathrm{TiO}_{2}$ & 11.4 & 19.0 & 59.9 & 850 & 4 & 20 & 0.15 \\
\hline 158 & $\mathrm{Zn}$ & $\mathrm{Zr}$ & $\mathrm{Tb}$ & $\mathrm{TiO}_{2}$ & 12.9 & 34.4 & 37.6 & 850 & 2 & 10 & 0.70 \\
\hline 159 & $\mathrm{Zn}$ & $\mathrm{Tb}$ & $\mathrm{Hf}$ & $\mathrm{TiO}_{2}$ & 11.0 & 30.4 & 36.2 & 850 & 2 & 15 & 0.70 \\
\hline 160 & Y & Cs & $\mathrm{Ce}$ & $\mathrm{TiO}_{2}$ & 12.4 & 39.1 & 31.8 & 850 & 2 & 20 & 0.40 \\
\hline 161 & Y & $\mathrm{Nd}$ & $\mathrm{Tb}$ & $\mathrm{TiO}_{2}$ & 8.7 & 38.4 & 22.6 & 850 & 2 & 20 & 0.15 \\
\hline 162 & Mo & $\mathrm{Ba}$ & $\mathrm{Ce}$ & $\mathrm{TiO}_{2}$ & 15.4 & 41.3 & 37.4 & 850 & 2 & 10 & 0.70 \\
\hline 163 & Mo & $\mathrm{Ce}$ & $\mathrm{Nd}$ & $\mathrm{TiO}_{2}$ & 7.0 & 31.9 & 21.9 & 900 & 2 & 20 & 0.70 \\
\hline 164 & Mo & $\mathrm{Ce}$ & W & $\mathrm{TiO}_{2}$ & 8.7 & 34.4 & 25.4 & 850 & 2 & 20 & 0.15 \\
\hline 165 & Cs & $\mathrm{Ba}$ & $\mathrm{Eu}$ & $\mathrm{TiO}_{2}$ & 13.3 & 39.3 & 33.9 & 800 & 2 & 20 & 0.15 \\
\hline 166 & $\mathrm{La}$ & $\mathrm{Ce}$ & $\mathrm{W}$ & $\mathrm{TiO}_{2}$ & 12.5 & 40.4 & 31.0 & 850 & 2 & 20 & 0.15 \\
\hline 167 & $\mathrm{Ce}$ & $\mathrm{Nd}$ & $\mathrm{Tb}$ & $\mathrm{TiO}_{2}$ & 10.8 & 34.9 & 31.0 & 800 & 2 & 15 & 0.40 \\
\hline 168 & $\mathrm{Li}$ & $\mathrm{Ca}$ & $\mathrm{Ca}$ & $\mathrm{ZrO}_{2}$ & 1.3 & 36.4 & 3.7 & 850 & 2 & 20 & 0.15 \\
\hline 169 & $\mathrm{Li}$ & Mo & $\mathrm{Nd}$ & $\mathrm{ZrO}_{2}$ & 16.7 & 38.3 & 43.5 & 700 & 2 & 20 & 0.40 \\
\hline 170 & $\mathrm{Li}$ & $\mathrm{Ba}$ & $\mathrm{Nd}$ & $\mathrm{ZrO}_{2}$ & 1.1 & 27.9 & 4.1 & 900 & 4 & 20 & 0.70 \\
\hline 171 & $\mathrm{Li}$ & $\mathrm{La}$ & $\mathrm{Eu}$ & $\mathrm{ZrO}_{2}$ & 6.3 & 31.3 & 20.1 & 800 & 2 & 20 & 0.15 \\
\hline 172 & $\mathrm{Li}$ & $\mathrm{La}$ & $\mathrm{W}$ & $\mathrm{ZrO}_{2}$ & 13.6 & 40.1 & 34.0 & 850 & 2 & 20 & 0.70 \\
\hline 173 & $\mathrm{Li}$ & Co & Y & $\mathrm{ZrO}_{2}$ & 2.2 & 26.0 & 8.5 & 750 & 2 & 10 & 0.15 \\
\hline 174 & $\mathrm{Li}$ & $\mathrm{Tb}$ & none & $\mathrm{ZrO}_{2}$ & 6.0 & 36.8 & 16.3 & 900 & 2 & 20 & 0.15 \\
\hline 175 & $\mathrm{Li}$ & $\mathrm{K}$ & $\mathrm{Mn}$ & $\mathrm{ZrO}_{2}$ & 10.7 & 38.0 & 28.1 & 750 & 2 & 15 & 0.15 \\
\hline 176 & $\mathrm{Na}$ & $\mathrm{Ba}$ & $\mathrm{Nd}$ & $\mathrm{ZrO}_{2}$ & 10.5 & 31.2 & 33.8 & 750 & 2 & 10 & 0.15 \\
\hline 177 & $\mathrm{Na}$ & $\mathrm{Eu}$ & W & $\mathrm{ZrO}_{2}$ & 18.2 & 40.8 & 44.6 & 850 & 2 & 20 & 0.70 \\
\hline 178 & $\mathrm{Na}$ & $\mathrm{V}$ & Mo & $\mathrm{ZrO}_{2}$ & 8.2 & 31.5 & 26.1 & 800 & 2 & 10 & 0.15 \\
\hline 179 & $\mathrm{Na}$ & $\mathrm{K}$ & none & $\mathrm{ZrO}_{2}$ & 6.4 & 38.7 & 16.6 & 750 & 2 & 20 & 0.15 \\
\hline 180 & $\mathrm{Mg}$ & $\mathrm{V}$ & $\mathrm{Hf}$ & $\mathrm{ZrO}_{2}$ & 3.2 & 33.7 & 9.4 & 850 & 2 & 20 & 0.15 \\
\hline
\end{tabular}




\begin{tabular}{|c|c|c|c|c|c|c|c|c|c|c|c|}
\hline 181 & $\mathrm{Mg}$ & $\mathrm{Zr}$ & W & $\mathrm{ZrO}_{2}$ & 9.6 & 26.8 & 35.9 & 850 & 2 & 10 & 0.70 \\
\hline 182 & $\mathrm{~K}$ & $\mathrm{Ba}$ & $\mathrm{Nd}$ & $\mathrm{ZrO}_{2}$ & 8.5 & 29.1 & 29.2 & 700 & 2 & 20 & 0.40 \\
\hline 183 & $\mathrm{~K}$ & $\mathrm{Mn}$ & $\mathrm{Zn}$ & $\mathrm{ZrO}_{2}$ & 6.2 & 36.9 & 16.8 & 750 & 2 & 20 & 0.15 \\
\hline 184 & $\mathrm{~K}$ & $\mathrm{Mn}$ & $\mathrm{Ce}$ & $\mathrm{ZrO}_{2}$ & 6.6 & 25.6 & 25.8 & 800 & 2 & 10 & 0.15 \\
\hline 185 & $\mathrm{~K}$ & $\mathrm{Pd}$ & $\mathrm{Hf}$ & $\mathrm{ZrO}_{2}$ & n.d. & n.d. & n.d. & n.d. & n.d. & n.d. & n.d. \\
\hline 186 & $\mathrm{Ca}$ & $\mathrm{V}$ & $\mathrm{Cu}$ & $\mathrm{ZrO}_{2}$ & 7.0 & 26.8 & 26.2 & 800 & 2 & 10 & 0.15 \\
\hline 187 & $\mathrm{Ca}$ & $\mathrm{Ni}$ & $\mathrm{Cu}$ & $\mathrm{ZrO}_{2}$ & 6.3 & 35.6 & 17.8 & 750 & 2 & 20 & 0.15 \\
\hline 188 & $\mathrm{Ca}$ & Sr & $\mathrm{Zr}$ & $\mathrm{ZrO}_{2}$ & 10.4 & 33.6 & 31.1 & 700 & 2 & 20 & 0.40 \\
\hline 189 & $\mathrm{Ca}$ & $\mathrm{Cu}$ & $\mathrm{Hf}$ & $\mathrm{ZrO}_{2}$ & 1.9 & 31.5 & 6.1 & 800 & 2 & 20 & 0.15 \\
\hline 190 & $\mathrm{~V}$ & $\mathrm{Mn}$ & $\mathrm{W}$ & $\mathrm{ZrO}_{2}$ & 7.8 & 34.1 & 23.0 & 850 & 2 & 20 & 0.15 \\
\hline 191 & $\mathrm{Mn}$ & $\mathrm{Ba}$ & none & $\mathrm{ZrO}_{2}$ & 5.4 & 27.1 & 20.1 & 750 & 2 & 15 & 0.15 \\
\hline 192 & $\mathrm{Mn}$ & $\mathrm{Tb}$ & Hf & $\mathrm{ZrO}_{2}$ & 1.4 & 46.7 & 3.0 & 850 & 2 & 10 & 0.70 \\
\hline 193 & $\mathrm{Mn}$ & $\mathrm{Fe}$ & $\mathrm{Nd}$ & $\mathrm{ZrO}_{2}$ & 4.7 & 34.2 & 13.8 & 850 & 2 & 20 & 0.40 \\
\hline 194 & $\mathrm{Fe}$ & $\mathrm{Cu}$ & $\mathrm{Zr}$ & $\mathrm{ZrO}_{2}$ & 6.1 & 36.2 & 16.9 & 850 & 2 & 20 & 0.15 \\
\hline 195 & Co & $\mathrm{Ce}$ & $\mathrm{Nd}$ & $\mathrm{ZrO}_{2}$ & 1.2 & 25.9 & 4.7 & 800 & 6 & 15 & 0.70 \\
\hline 196 & Co & Sr & $\mathrm{Ba}$ & $\mathrm{ZrO}_{2}$ & 4.9 & 12.3 & 39.7 & 900 & 6 & 20 & 0.70 \\
\hline 197 & $\mathrm{Ni}$ & Cs & $\mathrm{Ba}$ & $\mathrm{ZrO}_{2}$ & 1.2 & 39.6 & 2.9 & 700 & 2 & 20 & 0.40 \\
\hline 198 & $\mathrm{Ni}$ & $\mathrm{Ba}$ & $\mathrm{Hf}$ & $\mathrm{ZrO}_{2}$ & 1.3 & 22.0 & 6.0 & 850 & 6 & 15 & 0.40 \\
\hline 199 & $\mathrm{Ni}$ & $\mathrm{Y}$ & $\mathrm{Eu}$ & $\mathrm{ZrO}_{2}$ & 1.0 & 25.9 & 3.9 & 800 & 6 & 20 & 0.70 \\
\hline 200 & $\mathrm{Zn}$ & $\mathrm{La}$ & $\mathrm{Ce}$ & $\mathrm{ZrO}_{2}$ & 3.4 & 35.0 & 9.8 & 900 & 2 & 20 & 0.15 \\
\hline 201 & $\mathrm{Y}$ & Mo & $\mathrm{Nd}$ & $\mathrm{ZrO}_{2}$ & 7.4 & 30.1 & 24.5 & 800 & 2 & 15 & 0.15 \\
\hline 202 & $\mathrm{Pd}$ & Cs & $\mathrm{Ba}$ & $\mathrm{ZrO}_{2}$ & n.d. & n.d. & n.d. & n.d. & n.d. & n.d. & n.d. \\
\hline 203 & $\mathrm{La}$ & $\mathrm{Eu}$ & W & $\mathrm{ZrO}_{2}$ & 4.8 & 36.7 & 13.1 & 850 & 2 & 20 & 0.40 \\
\hline 204 & $\mathrm{Li}$ & $\mathrm{Mg}$ & $\mathrm{Zr}$ & $\mathrm{BaO}$ & 18.6 & 38.6 & 48.2 & 800 & 2 & 20 & 0.70 \\
\hline 205 & $\mathrm{Li}$ & Co & $\mathrm{Zn}$ & $\mathrm{BaO}$ & 6.2 & 32.7 & 19.0 & 900 & 2 & 20 & 0.70 \\
\hline 206 & $\mathrm{Li}$ & Co & $\mathrm{Nd}$ & $\mathrm{BaO}$ & 7.1 & 17.8 & 39.9 & 900 & 4 & 20 & 0.15 \\
\hline 207 & $\mathrm{Li}$ & Co & $\mathrm{Eu}$ & $\mathrm{BaO}$ & 13.8 & 34.3 & 40.1 & 800 & 2 & 15 & 0.15 \\
\hline 208 & $\mathrm{Li}$ & $\mathrm{Zr}$ & Cs & $\mathrm{BaO}$ & 16.4 & 36.6 & 44.9 & 850 & 2 & 20 & 0.70 \\
\hline 209 & $\mathrm{Na}$ & $\mathrm{Ca}$ & $\mathrm{Mn}$ & $\mathrm{BaO}$ & 9.0 & 28.5 & 31.7 & 800 & 2 & 20 & 0.40 \\
\hline 210 & $\mathrm{Na}$ & $\mathrm{Fe}$ & $\mathrm{Ce}$ & $\mathrm{BaO}$ & 9.3 & 33.0 & 28.2 & 850 & 2 & 20 & 0.40 \\
\hline 211 & $\mathrm{Mg}$ & $\mathrm{K}$ & $\mathrm{Y}$ & $\mathrm{BaO}$ & 17.0 & 37.2 & 45.6 & 850 & 2 & 20 & 0.70 \\
\hline 212 & $\mathrm{Mg}$ & $\mathrm{V}$ & $\mathrm{Mn}$ & $\mathrm{BaO}$ & 10.2 & 32.6 & 31.3 & 750 & 2 & 20 & 0.15 \\
\hline 213 & $\mathrm{Mg}$ & $\mathrm{Mn}$ & $\mathrm{Ni}$ & $\mathrm{BaO}$ & 8.7 & 30.0 & 29.1 & 850 & 2 & 20 & 0.70 \\
\hline 214 & $\mathrm{Mg}$ & $\mathrm{Pd}$ & none & $\mathrm{BaO}$ & 0.8 & 45.3 & 1.8 & 900 & 6 & 15 & 0.70 \\
\hline 215 & $\mathrm{Mg}$ & $\mathrm{Ni}$ & $\mathrm{W}$ & $\mathrm{BaO}$ & 9.6 & 24.9 & 38.6 & 850 & 2 & 20 & 0.70 \\
\hline 216 & $\mathrm{~K}$ & $\mathrm{Ca}$ & $\mathrm{Zr}$ & $\mathrm{BaO}$ & 13.7 & 36.6 & 37.3 & 850 & 2 & 20 & 0.70 \\
\hline 217 & $\mathrm{~K}$ & $\mathrm{~V}$ & Mo & $\mathrm{BaO}$ & 18.5 & 32.9 & 56.3 & 850 & 2 & 20 & 0.70 \\
\hline 218 & $\mathrm{~K}$ & $\mathrm{Zr}$ & $\mathrm{La}$ & $\mathrm{BaO}$ & 17.5 & 37.3 & 47.0 & 800 & 2 & 15 & 0.70 \\
\hline 219 & $\mathrm{Ca}$ & $\mathrm{Mn}$ & Mo & $\mathrm{BaO}$ & 10.3 & 32.5 & 31.7 & 850 & 2 & 20 & 0.40 \\
\hline 220 & $\mathrm{Ca}$ & $\mathrm{Y}$ & $\mathrm{Zr}$ & $\mathrm{BaO}$ & 11.0 & 38.3 & 28.6 & 800 & 2 & 20 & 0.40 \\
\hline 221 & $\mathrm{Ca}$ & $\mathrm{W}$ & none & $\mathrm{BaO}$ & 6.2 & 20.9 & 29.7 & 800 & 2 & 15 & 0.40 \\
\hline 222 & $\mathrm{~V}$ & $\mathrm{Mn}$ & $\mathrm{Cu}$ & $\mathrm{BaO}$ & 9.9 & 34.2 & 29.1 & 850 & 2 & 15 & 0.15 \\
\hline 223 & V & $\mathrm{Fe}$ & none & $\mathrm{BaO}$ & 13.9 & 29.4 & 47.1 & 850 & 4 & 20 & 0.15 \\
\hline 224 & $\mathrm{~V}$ & $\mathrm{Zr}$ & $\mathrm{Eu}$ & $\mathrm{BaO}$ & 12.9 & 36.2 & 35.6 & 900 & 2 & 20 & 0.70 \\
\hline 225 & $\mathrm{Mn}$ & $\mathrm{Y}$ & $\mathrm{Hf}$ & $\mathrm{BaO}$ & 6.1 & 17.2 & 35.5 & 900 & 4 & 15 & 0.15 \\
\hline 226 & $\mathrm{Fe}$ & $\mathrm{Ba}$ & $\mathrm{La}$ & $\mathrm{BaO}$ & 14.1 & 34.6 & 40.8 & 850 & 2 & 20 & 0.70 \\
\hline 227 & Co & $\mathrm{Zn}$ & $\mathrm{Zr}$ & $\mathrm{BaO}$ & 12.4 & 36.2 & 34.4 & 900 & 2 & 20 & 0.70 \\
\hline 228 & $\mathrm{Zn}$ & $\mathrm{Hf}$ & none & $\mathrm{BaO}$ & 16.9 & 36.6 & 46.0 & 850 & 2 & 20 & 0.70 \\
\hline 229 & $\mathrm{Sr}$ & Mo & none & $\mathrm{BaO}$ & 21.2 & 37.0 & 57.4 & 850 & 2 & 20 & 0.70 \\
\hline 230 & $\mathrm{Sr}$ & $\mathrm{Ba}$ & $\mathrm{Hf}$ & $\mathrm{BaO}$ & 10.6 & 35.9 & 29.6 & 850 & 2 & 20 & 0.70 \\
\hline 231 & Mo & Cs & W & $\mathrm{BaO}$ & 20.2 & 43.2 & 46.7 & 850 & 2 & 20 & 0.70 \\
\hline 232 & $\mathrm{Ce}$ & $\mathrm{Nd}$ & Hf & $\mathrm{BaO}$ & 15.2 & 35.4 & 43.0 & 800 & 2 & 20 & 0.15 \\
\hline 233 & $\mathrm{Li}$ & $\mathrm{Fe}$ & $\mathrm{Ba}$ & $\mathrm{La}_{2} \mathrm{O}_{3}$ & 15.2 & 35.5 & 43.0 & 700 & 2 & 10 & 0.15 \\
\hline 234 & $\mathrm{Li}$ & $\mathrm{Y}$ & $\mathrm{Eu}$ & $\mathrm{La}_{2} \mathrm{O}_{3}$ & 15.4 & 35.9 & 42.8 & 700 & 2 & 15 & 0.15 \\
\hline 235 & $\mathrm{Li}$ & $\mathrm{Ba}$ & $\mathrm{La}$ & $\mathrm{La}_{2} \mathrm{O}_{3}$ & 15.0 & 37.8 & 39.6 & 750 & 2 & 20 & 0.40 \\
\hline 236 & $\mathrm{Li}$ & $\mathrm{Nd}$ & $\mathrm{Tb}$ & $\mathrm{La}_{2} \mathrm{O}_{3}$ & 10.3 & 30.6 & 33.8 & 700 & 2 & 10 & 0.15 \\
\hline 237 & $\mathrm{Na}$ & $\mathrm{Ca}$ & none & $\mathrm{La}_{2} \mathrm{O}_{3}$ & 15.4 & 33.5 & 45.9 & 700 & 2 & 20 & 0.15 \\
\hline 238 & $\mathrm{Na}$ & $\mathrm{Fe}$ & $\mathrm{Tb}$ & $\mathrm{La}_{2} \mathrm{O}_{3}$ & 7.5 & 31.7 & 23.6 & 850 & 2 & 10 & 0.15 \\
\hline 239 & $\mathrm{Na}$ & $\mathrm{Pd}$ & W & $\mathrm{La}_{2} \mathrm{O}_{3}$ & 1.3 & 37.5 & 3.5 & 900 & 4 & 15 & 0.70 \\
\hline 240 & $\mathrm{Mg}$ & $\mathrm{K}$ & $\mathrm{Fe}$ & $\mathrm{La}_{2} \mathrm{O}_{3}$ & 11.1 & 19.7 & 56.3 & 750 & 4 & 20 & 0.15 \\
\hline 241 & $\mathrm{Mg}$ & $\mathrm{Ca}$ & $\mathrm{Nd}$ & $\mathrm{La}_{2} \mathrm{O}_{3}$ & 16.2 & 39.1 & 41.3 & 750 & 2 & 20 & 0.40 \\
\hline 242 & $\mathrm{~K}$ & Co & $\mathrm{Ce}$ & $\mathrm{La}_{2} \mathrm{O}_{3}$ & 2.4 & 24.4 & 9.7 & 800 & 2 & 15 & 0.15 \\
\hline
\end{tabular}




\begin{tabular}{|c|c|c|c|c|c|c|c|c|c|c|c|}
\hline 243 & $\mathrm{~K}$ & $\mathrm{Cu}$ & none & $\mathrm{La}_{2} \mathrm{O}_{3}$ & 7.5 & 38.4 & 19.6 & 850 & 2 & 20 & 0.15 \\
\hline 244 & $\mathrm{~K}$ & none & none & $\mathrm{La}_{2} \mathrm{O}_{3}$ & 7.9 & 29.4 & 27.0 & 800 & 2 & 10 & 0.15 \\
\hline 245 & $\mathrm{Ca}$ & $\mathrm{Mn}$ & $\mathrm{Sr}$ & $\mathrm{La}_{2} \mathrm{O}_{3}$ & 10.6 & 32.0 & 33.2 & 700 & 2 & 20 & 0.15 \\
\hline 246 & $\mathrm{Ca}$ & $\mathrm{Fe}$ & $\mathrm{Tb}$ & $\mathrm{La}_{2} \mathrm{O}_{3}$ & 12.3 & 32.3 & 38.0 & 750 & 2 & 10 & 0.15 \\
\hline 247 & $\mathrm{Ca}$ & $\mathrm{Pd}$ & $\mathrm{Tb}$ & $\mathrm{La}_{2} \mathrm{O}_{3}$ & n.d. & n.d. & n.d. & n.d. & n.d. & n.d. & n.d. \\
\hline 248 & $\mathrm{Ti}$ & Co & $\mathrm{Cu}$ & $\mathrm{La}_{2} \mathrm{O}_{3}$ & 1.3 & 18.3 & 7.1 & 900 & 6 & 20 & 0.15 \\
\hline 249 & $\mathrm{Ti}$ & Co & $\mathrm{Pd}$ & $\mathrm{La}_{2} \mathrm{O}_{3}$ & 0.6 & 40.6 & 1.5 & 700 & 2 & 20 & 0.40 \\
\hline 250 & $\mathrm{Ti}$ & $\mathrm{Zr}$ & $\mathrm{Ba}$ & $\mathrm{La}_{2} \mathrm{O}_{3}$ & 4.1 & 33.1 & 12.4 & 700 & 2 & 20 & 0.15 \\
\hline 251 & $\mathrm{Ti}$ & $\mathrm{Ni}$ & $\mathrm{Hf}$ & $\mathrm{La}_{2} \mathrm{O}_{3}$ & 9.6 & 36.4 & 26.4 & 900 & 2 & 20 & 0.70 \\
\hline 252 & $\mathrm{~V}$ & $\mathrm{Fe}$ & Mo & $\mathrm{La}_{2} \mathrm{O}_{3}$ & 7.7 & 34.2 & 22.5 & 800 & 2 & 20 & 0.15 \\
\hline 253 & V & Mo & none & $\mathrm{La}_{2} \mathrm{O}_{3}$ & 14.9 & 41.5 & 35.8 & 900 & 2 & 20 & 0.70 \\
\hline 254 & V & $\mathrm{La}$ & $\mathrm{W}$ & $\mathrm{La}_{2} \mathrm{O}_{3}$ & 14.1 & 34.6 & 40.8 & 850 & 2 & 20 & 0.70 \\
\hline 255 & $\mathrm{~V}$ & $\mathrm{Ce}$ & $\mathrm{Nd}$ & $\mathrm{La}_{2} \mathrm{O}_{3}$ & 8.4 & 30.6 & 27.3 & 700 & 2 & 20 & 0.15 \\
\hline 256 & $\mathrm{Mn}$ & $\mathrm{Sr}$ & Mo & $\mathrm{La}_{2} \mathrm{O}_{3}$ & 10.8 & 29.3 & 36.9 & 700 & 2 & 15 & 0.15 \\
\hline 257 & $\mathrm{Fe}$ & $\mathrm{Cu}$ & $\mathrm{Zr}$ & $\mathrm{La}_{2} \mathrm{O}_{3}$ & 4.9 & 33.0 & 14.7 & 850 & 2 & 20 & 0.15 \\
\hline 258 & $\mathrm{Fe}$ & $\mathrm{Nd}$ & $\mathrm{Tb}$ & $\mathrm{La}_{2} \mathrm{O}_{3}$ & 13.4 & 32.3 & 41.5 & 700 & 2 & 10 & 0.15 \\
\hline 259 & $\mathrm{Ni}$ & Hf & none & $\mathrm{La}_{2} \mathrm{O}_{3}$ & 0.9 & 41.2 & 2.2 & 750 & 2 & 20 & 0.40 \\
\hline 260 & $\mathrm{Cu}$ & Mo & $\mathrm{Pd}$ & $\mathrm{La}_{2} \mathrm{O}_{3}$ & 0.5 & 28.2 & 1.8 & 900 & 6 & 20 & 0.70 \\
\hline 261 & $\mathrm{Cu}$ & $\mathrm{La}$ & W & $\mathrm{La}_{2} \mathrm{O}_{3}$ & 8.2 & 37.7 & 21.6 & 850 & 2 & 20 & 0.15 \\
\hline 262 & $\mathrm{Zn}$ & Y & none & $\mathrm{La}_{2} \mathrm{O}_{3}$ & 15.1 & 40.9 & 36.9 & 750 & 2 & 20 & 0.15 \\
\hline 263 & $\mathrm{Zn}$ & $\mathrm{Zr}$ & Cs & $\mathrm{La}_{2} \mathrm{O}_{3}$ & 12.2 & 38.0 & 32.1 & 750 & 2 & 20 & 0.15 \\
\hline 264 & $\mathrm{Sr}$ & $\mathrm{Y}$ & $\mathrm{Ce}$ & $\mathrm{La}_{2} \mathrm{O}_{3}$ & 14.8 & 37.1 & 39.9 & 700 & 2 & 15 & 0.15 \\
\hline 265 & Mo & Cs & $\mathrm{Tb}$ & $\mathrm{La}_{2} \mathrm{O}_{3}$ & 13.3 & 30.1 & 44.2 & 700 & 2 & 10 & 0.15 \\
\hline 266 & $\mathrm{Pd}$ & $\mathrm{Ce}$ & $\mathrm{Tb}$ & $\mathrm{La}_{2} \mathrm{O}_{3}$ & 1.1 & 30.8 & 3.6 & 900 & 6 & 10 & 0.70 \\
\hline 267 & $\mathrm{Ba}$ & $\mathrm{Ce}$ & Hf & $\mathrm{La}_{2} \mathrm{O}_{3}$ & 13.9 & 39.4 & 35.4 & 750 & 2 & 20 & 0.15 \\
\hline 268 & $\mathrm{La}$ & $\mathrm{Tb}$ & none & $\mathrm{La}_{2} \mathrm{O}_{3}$ & 7.8 & 29.9 & 26.2 & 750 & 2 & 20 & 0.15 \\
\hline 269 & $\mathrm{~Tb}$ & $\mathrm{Hf}$ & $\mathrm{W}$ & $\mathrm{La}_{2} \mathrm{O}_{3}$ & 15.2 & 41.7 & 36.5 & 750 & 2 & 20 & 0.15 \\
\hline 270 & $\mathrm{Na}$ & $\mathrm{K}$ & $\mathrm{V}$ & $\mathrm{CeO}_{2}$ & 6.6 & 34.3 & 19.4 & 850 & 2 & 20 & 0.15 \\
\hline 271 & $\mathrm{Na}$ & V & $\mathrm{Y}$ & $\mathrm{CeO}_{2}$ & 7.3 & 38.3 & 19.1 & 850 & 2 & 20 & 0.15 \\
\hline 272 & $\mathrm{Mg}$ & $\mathrm{K}$ & none & $\mathrm{CeO}_{2}$ & 8.5 & 28.8 & 29.6 & 800 & 2 & 10 & 0.15 \\
\hline 273 & $\mathrm{Mg}$ & $\mathrm{Fe}$ & none & $\mathrm{CeO}_{2}$ & 7.0 & 27.5 & 25.5 & 800 & 2 & 10 & 0.15 \\
\hline 274 & $\mathrm{Mg}$ & $\mathrm{Ni}$ & $\mathrm{Zn}$ & $\mathrm{CeO}_{2}$ & 1.3 & 26.7 & 4.9 & 800 & 6 & 20 & 0.15 \\
\hline 275 & $\mathrm{~K}$ & Co & $\mathrm{Sr}$ & $\mathrm{CeO}_{2}$ & 6.4 & 32.8 & 19.5 & 900 & 2 & 20 & 0.40 \\
\hline 276 & K & $\mathrm{La}$ & $\mathrm{Ce}$ & $\mathrm{CeO}_{2}$ & 13.4 & 32.3 & 41.5 & 700 & 2 & 10 & 0.15 \\
\hline 277 & $\mathrm{~K}$ & $\mathrm{Ce}$ & $\mathrm{Eu}$ & $\mathrm{CeO}_{2}$ & 5.1 & 34.4 & 14.8 & 900 & 2 & 20 & 0.40 \\
\hline 278 & $\mathrm{Ti}$ & $\mathrm{Ni}$ & $\mathrm{Ba}$ & $\mathrm{CeO}_{2}$ & 4.8 & 36.2 & 13.2 & 750 & 2 & 15 & 0.70 \\
\hline 279 & $\mathrm{Ti}$ & $\mathrm{Zn}$ & $\mathrm{Pd}$ & $\mathrm{CeO}_{2}$ & 1.2 & 26.0 & 4.6 & 800 & 2 & 15 & 0.15 \\
\hline 280 & V & $\mathrm{Sr}$ & W & $\mathrm{CeO}_{2}$ & 1.7 & 24.6 & 7.0 & 850 & 6 & 20 & 0.40 \\
\hline 281 & $\mathrm{~V}$ & $\mathrm{Zr}$ & $\mathrm{Nd}$ & $\mathrm{CeO}_{2}$ & 7.5 & 35.5 & 21.1 & 850 & 2 & 20 & 0.15 \\
\hline 282 & $\mathrm{~V}$ & $\mathrm{Pd}$ & $\mathrm{W}$ & $\mathrm{CeO}_{2}$ & 1.6 & 42.9 & 3.7 & 800 & 2 & 10 & 0.70 \\
\hline 283 & $\mathrm{Mn}$ & $\mathrm{Ni}$ & $\mathrm{Zn}$ & $\mathrm{CeO}_{2}$ & n.d. & n.d. & n.d. & n.d. & n.d. & n.d. & n.d. \\
\hline 284 & $\mathrm{Mn}$ & $\mathrm{Ni}$ & $\mathrm{Y}$ & $\mathrm{CeO}_{2}$ & 2.9 & 32.2 & 9.1 & 900 & 2 & 20 & 0.40 \\
\hline 285 & $\mathrm{Mn}$ & $\mathrm{Sr}$ & $\mathrm{La}$ & $\mathrm{CeO}_{2}$ & 8.6 & 35.0 & 24.7 & 850 & 2 & 20 & 0.15 \\
\hline 286 & $\mathrm{Mn}$ & $\mathrm{Y}$ & $\mathrm{Zr}$ & $\mathrm{CeO}_{2}$ & 6.6 & 26.8 & 24.8 & 800 & 2 & 10 & 0.15 \\
\hline 287 & $\mathrm{Mn}$ & $\mathrm{Ba}$ & $\mathrm{La}$ & $\mathrm{CeO}_{2}$ & 8.6 & 34.9 & 24.7 & 850 & 2 & 20 & 0.15 \\
\hline 288 & $\mathrm{Mn}$ & none & none & $\mathrm{CeO}_{2}$ & 4.9 & 28.7 & 17.1 & 800 & 2 & 20 & 0.15 \\
\hline 289 & $\mathrm{Mn}$ & $\mathrm{Eu}$ & Hf & $\mathrm{CeO}_{2}$ & 6.2 & 35.1 & 17.7 & 800 & 2 & 20 & 0.15 \\
\hline 290 & $\mathrm{Fe}$ & $\mathrm{Zn}$ & $\mathrm{La}$ & $\mathrm{CeO}_{2}$ & 3.5 & 24.1 & 14.7 & 800 & 2 & 10 & 0.15 \\
\hline 291 & $\mathrm{Fe}$ & Y & $\mathrm{La}$ & $\mathrm{CeO}_{2}$ & 5.1 & 25.9 & 19.5 & 800 & 2 & 10 & 0.15 \\
\hline 292 & $\mathrm{Ni}$ & $\mathrm{Sr}$ & $\mathrm{Nd}$ & $\mathrm{CeO}_{2}$ & 10.2 & 34.4 & 29.8 & 800 & 2 & 20 & 0.40 \\
\hline 293 & $\mathrm{Ni}$ & $\mathrm{Nd}$ & $\mathrm{Eu}$ & $\mathrm{CeO}_{2}$ & 8.3 & 32.1 & 25.9 & 900 & 2 & 20 & 0.70 \\
\hline 294 & $\mathrm{Zn}$ & $\mathrm{Pd}$ & $\mathrm{Ce}$ & $\mathrm{CeO}_{2}$ & 2.2 & 32.3 & 6.8 & 800 & 2 & 20 & 0.15 \\
\hline 295 & $\mathrm{Zn}$ & $\mathrm{La}$ & $\mathrm{Nd}$ & $\mathrm{CeO}_{2}$ & 9.2 & 27.9 & 32.9 & 750 & 2 & 10 & 0.15 \\
\hline 296 & $\mathrm{Sr}$ & Cs & none & $\mathrm{CeO}_{2}$ & 12.5 & 36.1 & 34.8 & 800 & 2 & 20 & 0.40 \\
\hline 297 & $\mathrm{Sr}$ & $\mathrm{Ce}$ & none & $\mathrm{CeO}_{2}$ & 10.9 & 33.9 & 32.1 & 700 & 2 & 20 & 0.15 \\
\hline 298 & $\mathrm{Y}$ & $\mathrm{Zr}$ & $\mathrm{Eu}$ & $\mathrm{CeO}_{2}$ & 7.2 & 35.6 & 20.2 & 850 & 2 & 20 & 0.15 \\
\hline 299 & Mo & $\mathrm{Pd}$ & $\mathrm{Ba}$ & $\mathrm{CeO}_{2}$ & 1.4 & 45.3 & 3.0 & 850 & 2 & 20 & 0.70 \\
\hline 300 & Cs & $\mathrm{Ce}$ & $\mathrm{Nd}$ & $\mathrm{CeO}_{2}$ & 5.9 & 30.7 & 19.1 & 800 & 2 & 15 & 0.15 \\
\hline
\end{tabular}

${ }^{a}$ The catalyst composition is expressed in the form of M1-M2-M3/Support. The three active elements (M1-M3) are sorted along the atomic number. 
${ }^{b}$ The best $C_{2}$ yield of individual catalysts is reported together with the corresponding parameters. The performance of 9 catalysts was not determined due to sintering at the calcination temperature. The corresponding cells are filled with "n.d."

'The reaction in the absence of catalysts led to the $\mathrm{C}_{2}$ yield of $10.2 \%$ with the corresponding $\mathrm{CH}_{4}$ conversion of $29.7 \%$ and the $\mathrm{C}_{2}$ selectivity of $34.3 \%$ at the temperature of $850{ }^{\circ} \mathrm{C}$, the $\mathrm{CH}_{4} / \mathrm{O}_{2}$ ratio of $2 \mathrm{~mol} / \mathrm{mol}$, the total flow of $20 \mathrm{~mL} / \mathrm{min}$, and $\mathrm{P}_{\mathrm{Ar}}$ of $0.15 \mathrm{~atm}$. 

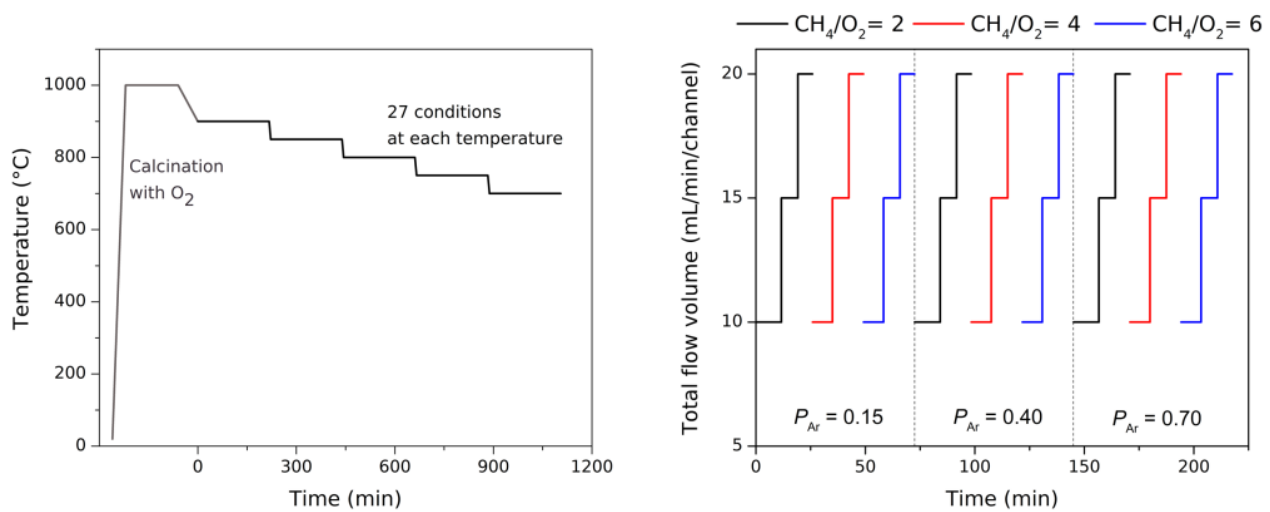

Figure S1. Programmed sequence of reaction conditions. Each temperature step includes a program for the gas flow.
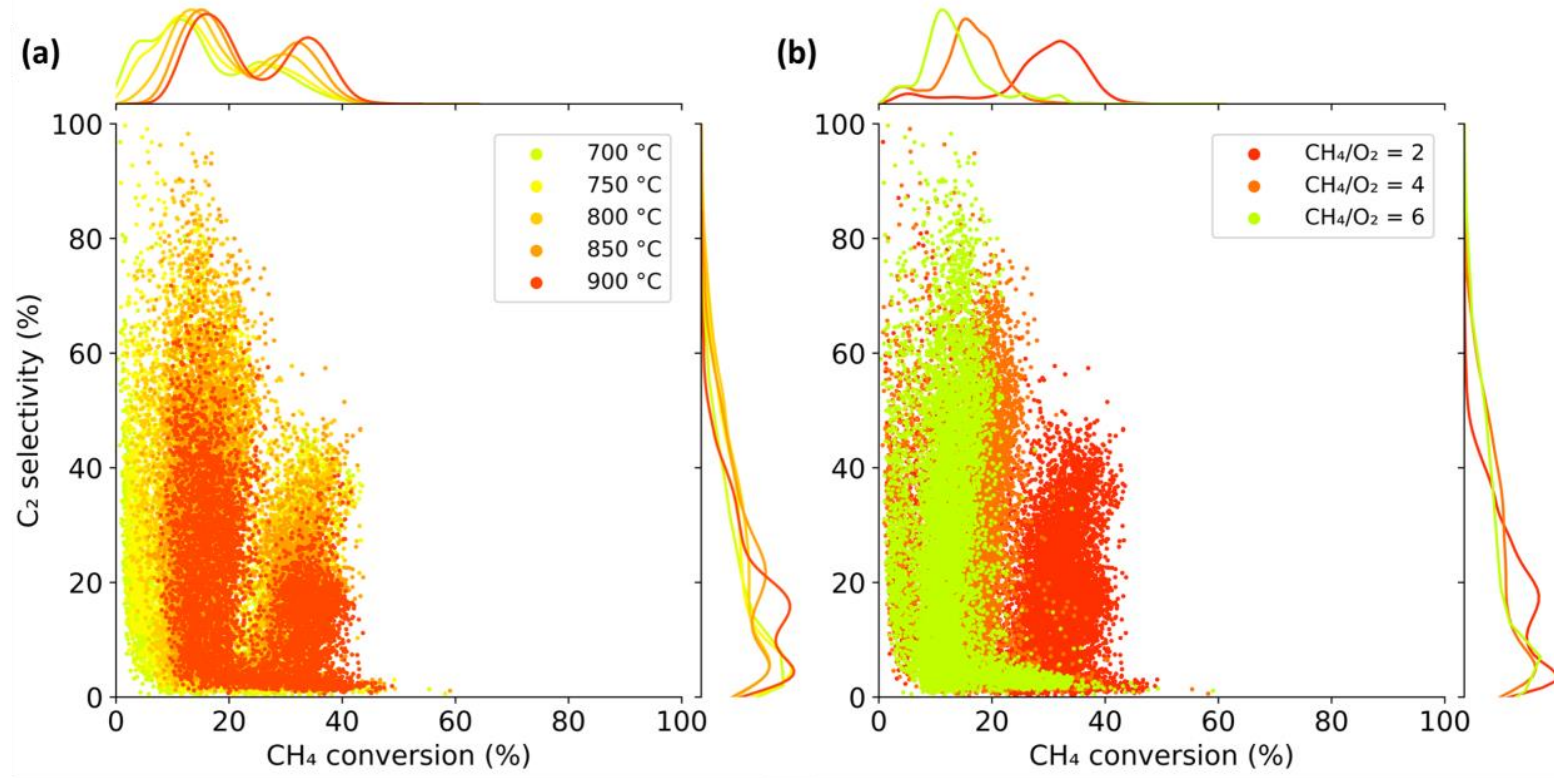

Figure S2. Scatter plots of $\mathrm{CH}_{4}$ conversion vs. $\mathrm{C}_{2}$ selectivity in terms of (a) the temperature and (b) the $\mathrm{CH}_{4} / \mathrm{O}_{2}$ ratio. 


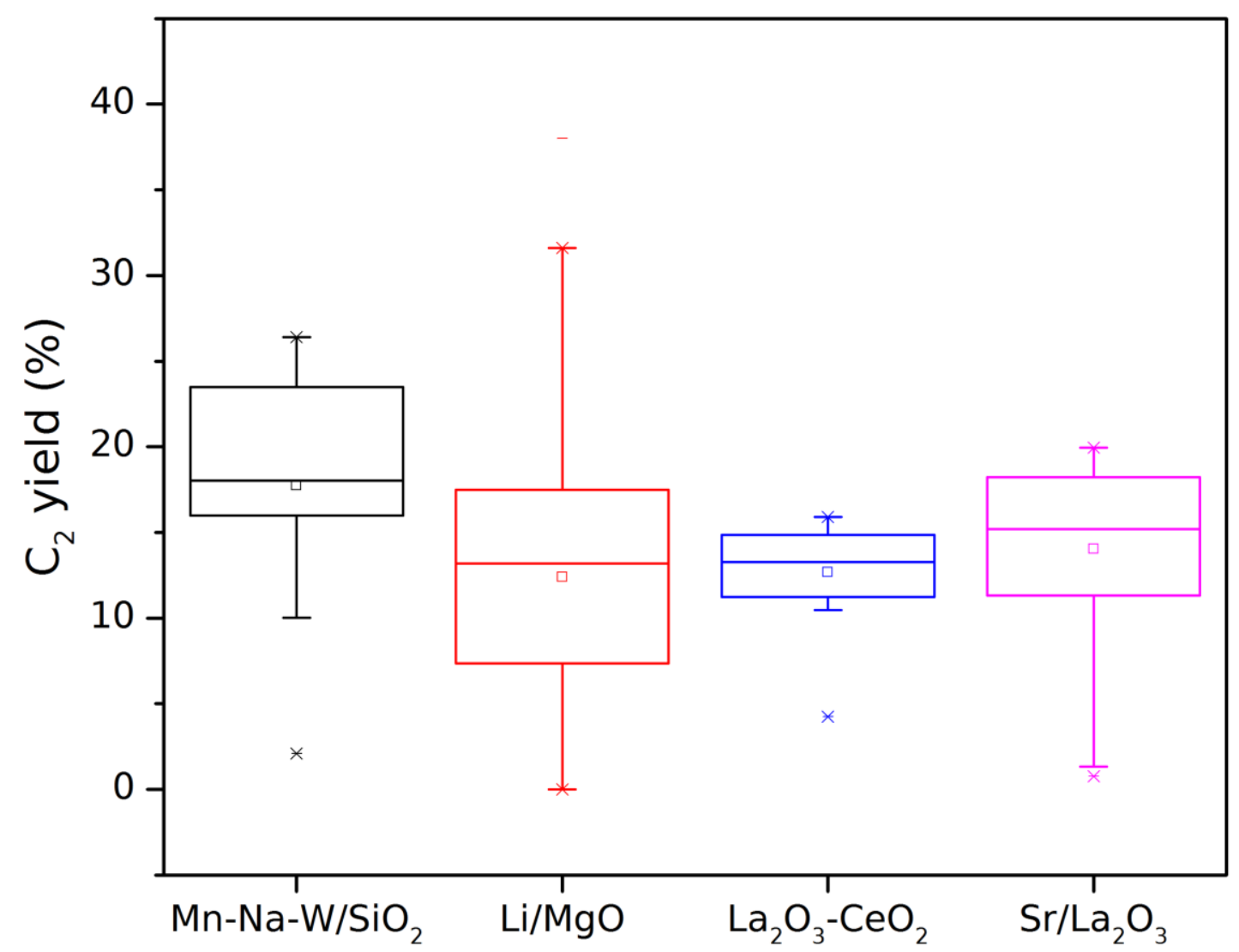

Figure S3. Box-and-whisker plot of the $\mathrm{C}_{2}$ yield for representative catalysts. The data are taken from a literature OCM data set collected by Zavyalova et al. ${ }^{37}$ 


\section{Table S2. List of descriptors}

\begin{tabular}{ll}
\hline Type & Descriptor \\
\hline Element (categorial) & $\mathrm{Li}, \mathrm{Na}, \mathrm{Mg}, \mathrm{K}, \mathrm{Ca}, \mathrm{Ti}, \mathrm{V}, \mathrm{Mn}, \mathrm{Fe}, \mathrm{Co}, \mathrm{Ni}, \mathrm{Cu}, \mathrm{Zn}, \mathrm{Sr}, \mathrm{Y}, \mathrm{Zr}, \mathrm{Mo}, \mathrm{Pd}, \mathrm{Cs}, \mathrm{Ba}, \mathrm{La}, \mathrm{Ce}, \mathrm{Nd}, \mathrm{Eu}, \mathrm{Tb}, \mathrm{Hf}, \mathrm{W}$ \\
Support (categorial) & $\mathrm{MgO}_{2} \mathrm{Al}_{2} \mathrm{O}_{3}, \mathrm{SiO}, \mathrm{CaO}, \mathrm{TiO}, \mathrm{ZrO}, \mathrm{BaO}, \mathrm{La}_{2} \mathrm{O}_{3}, \mathrm{CeO}_{2}$ \\
Group (categorial) & $\mathrm{G} 1, \mathrm{G} 2, \mathrm{G} 3, \mathrm{G} 4, \mathrm{G} 5, \mathrm{G} 6, \mathrm{G} 7, \mathrm{G} 8, \mathrm{G} 9, \mathrm{G} 10, \mathrm{G} 11, \mathrm{G} 12, \mathrm{GLa}$ \\
Atomic (numerical) & $\begin{array}{l}\max (x), \min (x), \operatorname{mean}(x): x=\text { electronegativity, electron affinity, 1st ionization energy, atomic radius, } \\
\text { atomic number }\end{array}$ \\
\hline
\end{tabular}

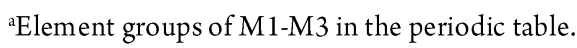

${ }^{\mathrm{b}}$ Maximum, minimum, and mean values of specified atomic features among M1-M3. 